\title{
On expressing vague quantification and scalar implicatures in the logic of partial information
}

\author{
Areski Nait Abdallah ${ }^{1}$, Alain Lecomte ${ }^{23}$ \\ 1 INRIA-Rocquencourt, France \\ 2 Université de Grenoble, CLIPS-IMAG \\ ${ }^{3}$ Université de Bordeaux, équipe SIGNES, LaBRI-INRIA, France
}

\begin{abstract}
In this paper, we use the logic of partial information to re-examine some early analyses of vague quantifiers in French such as quelques, peu, beaucoup that are found in particular in the work of $\mathrm{O}$. Ducrot [2]. Our approach is based on the paradigm offered by the logical formalization of the sorites paradox. We claim that this paradox offers a general scheme along which the argumentation structure of all vague quantifiers in French may be expressed. We offer a variational principle approximating Grice's maxims in the case of vague quantification.
\end{abstract}

Keywords: vague quantification, implicatures, argumentation scales, partial information.

\section{Introduction}

Most natural language quantifiers are vague. In this paper we are interested in vague quantification in the French language, although our methods may be generalized to other languages. In French, some examples of vague quantifiers are given by beaucoup, peu, la plupart, quelques, pas tous, etc. They all express partial information, and as such, their adequate treatment falls under the scope of a logic of partial information [4]. Such a logic should allow the correct inferences expected from linguistic usage to be drawn formally. These inferences are of more than one kind. For example, from

$$
\text { il a lu quelques romans de Balzac }
$$

(he has read some novels of Balzac) one should be able to trivially infer:

$$
\text { il a lu au moins un roman de Balzac }
$$

(he has read at least one novel of Balzac.) Such a consequence literally follows from the sentence uttered: we shall say that it is "hard" consequence of the sentence in the sense that it is impossible to question such a consequence, once the statement of the sentence from which it follows has been accepted. But one should also be able to deduce (first pointed out by [3], see also [1]):

$$
\text { il n'a pas lu quelques romans de Balzac }
$$


(he has not read some novels of Balzac). Such a step is usually justified by calling up Grice's maxims : if it were not the case, namely if the person spoken about had read all of Balzac novels, and if the speaker had been aware of that fact, then he would not have uttered (1), but rather

$$
\text { il a lu tous les romans de Balzac }
$$

The latter inference, however, does not have the same "firmness" as (2), as later in the discourse one may find a retraction such as

$$
\text { il les a même tous lus }
$$

(he has even read all of them.) According to [2], and in a manner that we feel is similar, one should be able to infer:

$$
\text { il connaît donc un peu Balzac }
$$

(he knows a bit about Balzac.) This situation differs from sentence

$$
\text { il n'a pas lu tous les romans de Balzac }
$$

(he has not read all of Balzac novels) which, although apparently inducing that the person under consideration has read more novels that the one spoken about in (1), is preferentially oriented towards the tentative conclusion:

$$
\text { il ne connaît donc pas parfaitement Balzac }
$$

(he does not have a perfect knowledge of Balzac.) It should be noted however, that these two conclusions are defeasible, in the sense that we very well may have fragments of discourse analogous to the following:

il a lu quelques romans de Balzac, pourtant il ne connaît pas parfaitement Balzac

(he has read some of Balzac novel, still he does not have a perfect knowledge of Balzac.) or

$$
\text { il a lu quelques romans de Balzac, pourtant il connâ̂t un peu Balzac }
$$

(he has read some of Balzac novels, still he does have some knowledge of Balzac.) In contrast, it must be noted that it is impossible to write (11)

*il a lu quelques romans de Balzac, pourtant (ou même, etc.) il n'en a lu aucun.

(he has read some of Balzac novels, still (or even, etc.) he has read none.) This means that the conclusions drawn in (3) and (6) do not have the same status as those drawn in (2). Such defeasible conclusions will be called weak. The aim of the logic of Partial Information is to provide an account of both hard and weak inference types. In that sense we think its application to the issue of scalar implicatures is legitimate. 


\section{Logic of partial information (LPI)}

We briefly sketch the major ideas of the logic of partial information [4]. The logic may be seen as an extension of classical partial logic, with two truth values 0,1 and an absence of truth value denoted by $\perp$. Kleene's strong tables for propositional logic connectives will be preferably used.

\begin{tabular}{|c|c|c|c|}
\hline$\phi \wedge \psi$ & 1 & 0 & $\perp$ \\
\hline 1 & 1 & 0 & $\perp$ \\
\hline 0 & 0 & 0 & 0 \\
\hline$\perp$ & $\perp$ & 0 & $\perp$ \\
\hline
\end{tabular}

\begin{tabular}{|c|c|c|c|}
\hline$\phi \vee \psi$ & 1 & 0 & $\perp$ \\
\hline 1 & 1 & 1 & 1 \\
\hline 0 & 1 & 0 & $\perp$ \\
\hline$\perp$ & 1 & $\perp$ & $\perp$ \\
\hline
\end{tabular}

\begin{tabular}{|c|c|}
\hline$\phi$ & $\neg \phi$ \\
\hline 1 & 0 \\
\hline 0 & 1 \\
\hline$\perp$ & $\perp$ \\
\hline
\end{tabular}

\begin{tabular}{|c|c|c|c|}
\hline$\phi \rightarrow \psi$ & 1 & 0 & $\perp$ \\
\hline 1 & 1 & 0 & $\perp$ \\
\hline 0 & 1 & 1 & 1 \\
\hline$\perp$ & 1 & $\perp$ & $\perp$ \\
\hline
\end{tabular}

This yields a fragmented notion of truth in a partial model and of semantic scope. If $P$ is the set of propositional variables, $f$ a partial truth value assignment (also called valuation) $f: P \rightarrow\{0,1\}, \varphi$ a formula, one defines satisfaction in the classical sense as $f \models \varphi$ iff $f(\varphi)=1$, and potential satisfaction as $f \|=\varphi$ iff $f(\varphi) \neq 0$.

In order to fill the gaps of our partial knowledge by means of tentative knowledge, valuations are generalized to triples $\left(i_{0}, J, i_{1}\right)$ where $i_{0}$ and $i_{1}$ are valuations, and $J$ is a non-empty set of valuations, such that $i_{1}$ extends $i_{0}$, every $j \in J$ extends $i_{0}$. The basic schematic structure of ionic interpretations $\left(i_{0}, J, i_{1}\right)$ may be summarized as follows:

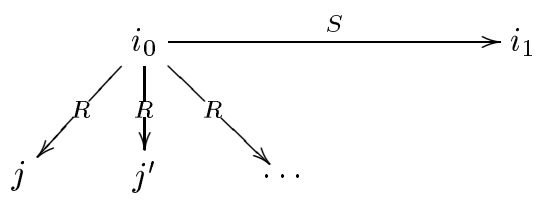

Valuation $i_{0}$ corresponds to hard knowledge, $i_{1}$ corresponds to soft, tentative knowledge, and set $J$ represents the justifications.

- $i_{0}$ is the kernel valuation (hard knowledge)

- $J=\left\{j, j^{\prime}, \ldots\right\} \neq \emptyset$ are potential extensions of $i_{0}$ (justification knowledge)

$-i_{1}=$ is the belt valuation (soft knowledge)

where

1. soft knowledge extends hard knowledge,

2. potential extensions extend hard knowledge: $i_{0} \sqsubseteq j$ for each $j \in J$

At the syntactic level, one introduces a new partial implication $\star(n, p)$, called a partial information ion. In essence $\star(n, p)$ is true iff justification $n$ is acceptable, and conclusion $p$ is true in a "soft" sense, or justification $n$ is not acceptable. 
The reader is referred to [4] for further details. The central points of relevance to this paper have to do with the following comparison of LPI versus non-monotonic reasoning:

1. Non-monotonic reasoning theories offer a global approach to ordinary commonsense reasoning; they specify coherent sets of beliefs.

2. In the standard approaches to non-monotonic reasoning, justifications are swept under the rug.

3. In a theory of implicitness, justifications cannot be swept under the rug.

4. Whence the need of a local approach managing stepwise all the elements of the reasoning process at each point.

5. It turns out that the non-monotonic character of commonsense reasoning is a secondary surface effect following from the fact that we always reason with partial information.

\section{The logic of partial information and the sand heap paradox}

The logic of partial information offers a paradigmatic way of treating imprecision, which illustrated by the famous sand heap paradox, due to Eubulides of Miletus. I start with a heap of sand containing $N$ grains of sand. I remove those grains one by one. I know that when there will be only a few grains left, there will be no heap left to be spoken about. What happens in-between these two extreme cases? Above all, when am I in a position to decide that there is no sand heap left? [4] p. 463 proposes to summarize the data as follows:

$$
\begin{aligned}
& \neg p(1) \\
& p(N) \\
& \forall n . p(n+1) \rightarrow \star(p(n), p(n))
\end{aligned}
$$

where $p(n)$ stands for property : $n$ grains of sand constitute a heap of sand. In this description, formula $\neg p(1)$ corresponds to situations where we are in a position to answer a definite hard no to the question Do we have a sand heap? It corresponds to the negative boundary of predicate $p$. Similarly, formula $p(N)$ corresponds to the case where we are in a position to answer a definite hard yes to the question Do we have a sand heap? It corresponds to the positive boundary of predicate $p$.

The third formula (14) expresses a sorites axiom, and should be understood as follows: for any $n$, if $n+1$ grains of sand constitute a heap of sand, then on the condition that we admit as a justification that $n$ grains of sand still constitute a heap, we will be able to deduce, albeit in a weak manner, that $n$ grains of sand constitute a heap. If we start with $N$ grains, which is the value for which we acknowledge that there is indeed a "heap of sand" and if we decrease $n$, then we must admit more and more justifications in order to infer the presence of a heap of sand. Furthermore, such a presence will only be inferred in a weaker and 
weaker form. One may assume that there is in this descent some value $n_{\max }$, such that $p\left(n_{\max }\right)$ is still an admissible justification, but not $p\left(n_{\max }-1\right)$. When $n$ ranges between $n_{\max }$ and 1 , the truth value of $p(n)$ is simply undetermined: this is in good correspondence with our intuition.

In this case we have only one sorites axiom, with a downward induction.

\section{An application to the case of quelques}

By using LPI, we get defeasability and the availability of justifications as computation objects for free. Remain to be solved the following two questions: What justifications should one accept in order to admit the truth of a sentence such as (1)? What is the computational meaning of Ducrot's orientation of the argumentation scale?

\subsection{Generalizing the sorites to vague quantifier quelques}

Let us start with the following formalization for a sentence containing quelques. Let

- $l u(k, n)$ stand for " $k$ a lu exactement $n$ romans de Balzac."

- heap $(k)$ for " $k$ a lu quelques romans de Balzac."

$-\operatorname{luq}(k, n):=(l u(k, n) \rightarrow$ heap $(k))$ for " $k$ a lu quelques romans de Balzac s'il en a lu $n "$

We assume that the semantic content of a sentence such as (3) is given by the following axioms:

(Q1) $\forall k l u(k, 0) \rightarrow \neg h e a p(k)$

(Q2) $\exists N \exists N^{\prime} N \leq N^{\prime} . \forall k \forall n . N \leq n \leq N^{\prime} \rightarrow l u q(k, n)$

$(q 2 b) \quad \forall k \forall n<N . \operatorname{luq}(k, n+1) \rightarrow \star(\operatorname{luq}(k, n), \operatorname{lu} q(k, n))$

$(q 2 c) \quad \forall k \forall n \geq N^{\prime} . \operatorname{lu} q(k, n) \rightarrow \star(\operatorname{luq}(k, n+1), \operatorname{lu} q(k, n+1))$

(Q3) The above reasons are the sole basis upon which one may claim predicate $h e a p(k)$ to hold.

The scope of the existential quantification $\exists N \exists N^{\prime}$ is the three univeral formulae in (Q2) through (q2c). These axioms will be part of the definition of quelques, in other words, we have a (hard) boundary part and a (weak) sorites part:

- negative boundary: it is not possible to claim on a lu quelques romans (one has read some novels) when one has not read any,

- positive boundary: there exists two bounds, a left bound $N$ and a right bound $N^{\prime}$, such that it is difficult to claim that on a lu quelques romans (one has read some novels), in the case one has read less than $N$ novels. It is also difficult to claim that on n'a lu que quelques romans (one has only read some novels) in the case one has read more than $N^{\prime}$ novels ....

- sorites: There exist two induction "principles" allowing one to extend hard information: 
- downward induction: it becomes increasingly difficult to admit that one has read some novels once one goes below left bound $N$ of the positive boundary. This is expressed by downward sorites axiom $(q 2 b)$.

- upward induction: it becomes increasingly difficult to admit that one has read some novels once one goes beyond right bound $N^{\prime}$ of the positive boundary. This is expressed by upward sorites axiom $(q 2 c)$.

Clause (Q1) is not expressible in terms of predicate $\operatorname{luq}(k, n)$, it is part of the definition of this predicate. This incites us to think that there is a dual definition associated with the definition of quelques namely the definition of pas ... quelques (corresponding in the current context to the construction il n'a pas lu quelques romans de Balzac (he has not read some novels of Balzac). Using abbreviations,

- nheap $(k)$ for " $k$ n'a pas lu quelques romans de Balzac."

- negluq $(k, n):=(\neg l u(k, n) \rightarrow$ nheap $(k))$ for " $k$ n'a pas lu quelques romans de Balzac s'il n'en a pas lu $n$ "

We assume that the semantic content of a sentence such as (1) is given by the following axioms:

$(\bar{Q} 1) \forall k l u(k, T) \rightarrow \neg$ nheap $(k)$

(Q 2$) \quad \exists M \exists M^{\prime} M<M^{\prime}$.

$(\bar{q} 2 a) \quad \forall k \forall n . M \leq n \leq M^{\prime} \rightarrow \operatorname{negluq}(k, n)$

$(\bar{q} 2 b) \quad \forall k \forall n<M . \operatorname{negluq}(k, n+1) \rightarrow \star(\operatorname{negluq}(k, n), \operatorname{negluq}(k, n))$

$(\bar{q} 2 c) \quad \forall k \forall n \geq M^{\prime} . \operatorname{negluq}(k, n) \rightarrow \star(\operatorname{negluq}(k, n+1), \operatorname{negluq}(k, n+1))$

$(\bar{Q} 3)$ The above reasons are the sole basis upon which one may claim predicate nheap $(k)$ to hold.

where $T$ is the total number of Balzac novels. Observe that the pair $\left(M, M^{\prime}\right)$ is not necessarily identical to the pair $\left(N, N^{\prime}\right)$ occurring in the first set of clauses, even though it might be.

Also observe that clauses (Q3) and ( $\bar{Q} 3)$ express closure properties. They permit a Prolog-like negation as finite failure to be defined: it is impossible to claim Pierre a lu quelques romans de Balzac (resp. Pierre n'a pas lu quelques romans de Balzac) unless such a claim follows from clauses (Q1) through (Q3) (resp. from clauses $(\bar{Q} 1)$ through $(\bar{Q} 3))$. In other words, “on n'a pas lu quelques romans de Balzac" (one has not read some of Balzac novels) may be claimed only if:

$-N \leq n \leq N^{\prime}$ and one has read $n$ such novels

- or $n<N, n \neq 0$ and one has some justifications for the claim

- or $n>N$, and one has some justifications for the claim.

These clauses are thus to be read as a logic program, and this point will be elaborated upon later. So, let us reconsider sentence (1) above il a lu quelques romans de Balzac. Such a sentence is valid (accepted) only if one falls into one of the three previous cases, namely:

- if $N \leq n \leq N^{\prime}$, then $l u(k, n) \rightarrow$ heap $(k)$, but $l u(k, n) \rightarrow \neg(l u(k, T-n)$. This yieds three subcases: 
- $M \leq T-n \leq M^{\prime}$, in which case one has nheap $(k)$

- $T-n<M$, in which case some justifications will be needed to establish concluation nheap $(k)$, which will be available as a weak conclusion.

- $T-n>M^{\prime}$, in which case some justifications will also be needed to establish weak conclusion nheap $(k)$. Since necessarily $n \neq 0$, one has $T-n \neq T$, which prevents from reaching $n h e a p(k)$ in the case $k$ has read all novels.

- if $n<N$, then some justifications are needed in order to prove heap $(k)$; for $n h e a p(k)$, however, such a proof will depend on the position of $T-n$ with respect to $M^{\prime}$, and so on.

- the case $n>N^{\prime}$ is similar.

Therefore, in all cases, from (1) one may deduce (3) (whether in a strong sense or in a weak sense.)

It thus appears that heap $(k)$ and nheap $(k)$ are not identical, if only because the former is irreducibly false whenever $l u(k, 0)$, whereas the same holds for the latter when $l u(k, T)$. We shall assume another difference that will best show their duality : the likelihood of heap $(k)$ decreases more drastically when the number $n$ of novels decreases on the short side of $N$ than when $n$ grows well beyond $N^{\prime}$, while the opposite holds for $n h e a p(k)$ (this will be made more precise later on). In such a case, we shall say that heap $(k)$ is positively oriented (i.e. in the direction of ascending induction), while nheap $(k)$ is negatively oriented (i.e. in the sense of descending induction). If we now admit that "the words of the discourse" ("les mots du discours" in Ducrot's expression) are sensitive with respect to this orientation, then we shall be able to explain why

il a lu quelques romans de Balzac, il les a même tous lus

(he has read some novels of Balzac, he has even read all of them) is permitted, whereas

$$
\text { *il a lu quelques romans de Balzac, il en a même lu un }
$$

(he has read some novels of Balzac, he has even read one of them) is not. We suggest positing the following hypothesis:

Même fits in the preferred orientation of the predicate associated with the scalar word

and even more precisely:

Même triggers the maximal inference possible in the preferred orientation of the predicate associated with the scalar word

\subsection{Evaluating softness degrees: natural deduction and logic programming}

We are now in a position to start addressing the question asked at the beginning of this Section. 
Let us assume that as a general rule, $k$ a lu quelques romans de Balzac s'il en lu $N_{1}$ ( $k$ has read some novels of Balzac if he has read $N_{1}$ such novels), expressed as $\forall k . l u q\left(k, N_{1}\right)$. Such a rule may be given by the context of the discourse under consideration. Let us also assume that la personne dont on parle en a effectivement lu $N_{2}$ (the person being spoken about has in fact read $N_{2}$ novels), expressed by $l u\left(c, N_{2}\right)$.

Let $N_{1}=5, N_{2}=3$. The goal is to establish the degree of softness of assertion il a lu quelques romans, knowing that the general rule is that $X$ a lu quelques romans de Balzac s'il en a lu cinq, expressed by $\forall k \cdot l u q(k, 5)$-and that "la personne dont on parle en a effectivement lu trois." expressed by $l u(c, 3) . "$ The corresponding natural deduction uses these two premisses as well as the first (downward) sorites axiom. It is as follows.

$$
\begin{aligned}
& \frac{\frac{\forall k . l u q(k, 5)}{\operatorname{lu} q(c, 5)} \forall \mathcal{E} \quad \forall k \forall n . l u q(k, n) \rightarrow \star(\operatorname{lu} q(k, n-1), \operatorname{lu} q(k, n-1))}{\star(\operatorname{lu} q(c, 4), \operatorname{lu} q(c, 4))} \\
& \frac{\frac{\star(\operatorname{luq}(c, 4), \star(\operatorname{lu} q(c, 3), \operatorname{lu} q(c, 3)))}{\star(\operatorname{lu} q(c, 4), \star(\operatorname{lu} q(c, 3), \operatorname{lu}(c, 3) \rightarrow \text { heap }(c)))}}{\frac{\star(\operatorname{lu} q(c, 4), \star(\operatorname{lu} q(c, 3), \text { heap }(c)))}{\exists j_{1}, \ldots, j_{s} . \star\left(j_{1}, \ldots, \star\left(j_{s}, \text { heap }(c)\right) \ldots\right)} \exists \mathcal{I}} \Rightarrow C \mathcal{E}
\end{aligned}
$$

Conclusion $\exists j_{1}, \ldots, j_{s} . \star\left(j_{1}, \ldots, \star\left(j_{s}\right.\right.$, heap $\left.\left.(c)\right) \ldots\right)$ corresponds to defeasible statement "Il a lu quelques romans de Balzac, sous reserve de l'acceptation des justifications $j "$ (he has read some novels of Balzac, subject to the acceptance of justifications $j$ ). This natural deduction calls upon hard fact $l u(c, 3)$, rule $\forall k . l u q(k, 5)$ and the downward sorites axiom. The models scheme having soft conclusion heap $(c)$ includes pattern $+\star l u q(c, 4),+\star l u q(c, 3)={ }_{\text {soft }}$ heap $(c)$

This conclusion is reminiscent of a logic programming query. As a matter of fact, if one starts with infinitary query (see [4] p. 507), heap $(c)^{\infty}$, the following logic programming derivation in LPI obtains:

$$
\begin{array}{ll}
\operatorname{heap}(c)^{\infty} \rightarrow & \\
(\operatorname{lu}(c, 3) \rightarrow \text { heap }(c))^{\infty} \rightarrow & \text { use fact } \operatorname{lu}(3, c) \\
(\operatorname{luq}(c, 3))^{\infty} \rightarrow & \text { use def. } \operatorname{lu} q(3, c) \\
(\operatorname{lu} q(c, 4))^{\infty} \rightarrow & \text { use justif. } \xi_{1}=\operatorname{lu} q(3, c) \\
(\operatorname{lu} q(c, 5))^{\infty} \rightarrow & \text { use justif. } \xi_{2}=\operatorname{lu} q(4, c) \\
()^{\infty} \rightarrow & \text { use axiom } \forall k . \operatorname{lu} q(k, 5), k=c
\end{array}
$$

This derivation ends with an empty query, and thus succeeds with justification answer substitution given by $\xi_{1}=\operatorname{lu} q(3, c), \xi_{2}=\operatorname{lu} q(4, c)$. These correspond to the justifications that must be accepted in order to derive initial goal $I l$ a $l u$ quelques romans de Balzac. 


\subsection{Topology of the argumentation scale}

It seems that an important property of the framework outlined here is the subdivision of the argumentation scale into three parts: the positive and negative boundaries, corresponding to the hard information part, and the sorites induction axioms part, corresponding to the cases where only soft conclusions may be drawn.

\subsubsection{Orientation of the argumentation scale and approximation of Grice's maxims}

The orientation of the argumentation scale in the sense of Ducrot may be expressed in our framework by saying that those justifications that aim at obtaining weak conclusions that are closer to the negative boundary have a cost, and that they cost more than justifications supporting a weak conclusion closer to the positive boundary. Hard conclusions have a zero cost.

More precisely, rule stated above:

Même triggers the maximal inference possible in the preferred orientation of the predicate associated with the scalar word

may be expressed by saying the following:

1. "maximal possible inference" may be understood as "draw a maximal set of conclusions"

2. "preferred orientation" : all predicate modified by a vague quantifier are oriented towards their positive boundary.

3. in other words, one will draw, as a set of possible conclusions, all those that follow from the positive boundary, plus what follows from the application of the sorites induction axioms.

4. in *il a lu quelques romans de Balzac, il en a même lu un, claim il a lu un has a maximal cost, according to the definition above.

5. therefore it must be discarded.

Technical details are as follows.

\subsubsection{Cost ordering}

One may posit as a general principle that

- Every piece of knowledge produced in the reasoning process has a cost.

- The aim of the reasoning process is to minimize the cost of the conclusions obtained.

Hard conclusions have cost zero. Soft conclusions that tend to the negative boundary have a higher cost that those tending to the positive boundary i.e. the likelihood of the corresponding conclusion decreases more drastically in the former case than in the latter. 
The cost of a conclusion is (the minimum possible value of) the total cost of all justifications supporting such a conclusion. More precisely, if $\Gamma$ is the current context, and $\varphi$ a claim:

$$
\begin{aligned}
\operatorname{cost}(\varphi)= & \text { cost of the shortest justification sequence } \psi_{1}, \ldots, \psi_{n} \text { such that } \\
& \Gamma \vdash \star\left(\psi_{1}, \star\left(\psi_{2}, \ldots \star\left(\psi_{n}, \varphi\right) \ldots\right)\right)
\end{aligned}
$$

Minimizing costs of conclusions amounts to

- spontaneously preferring hard conclusions over soft ones, and

- spontaneously preferring soft conclusions tending towards the positive boundary over soft conclusions tending towards the negative boundary.

Partial order on sets of beliefs may be only partially known.

We may even go further in formalisation, as seen in the next subsection.

\subsubsection{Paths, phase space and variational principle}

The notion of path (syntactic and semantic) is introduced in [4] chap. 19. Syntactically, a path is an increasing sequence of sets of partial information ionic formulae $\left(\Phi_{0}, \Phi_{1}, \ldots\right)$, semantically, it corresponds to a sequence of models $\left(m_{0}, m_{1}, \ldots, m_{n}, \ldots\right)$ such that for each $\mathrm{i}=0, \ldots, m_{i} \sqsubseteq m_{i+1}$ for the information ordering, $m_{i}=\Phi_{i}$ and the least upper bound $m$ of $\left(m_{n}\right)$ is a model of the least upper bound of the syntactic path. $\left(\Phi_{i}\right)_{i=0, \ldots}$ represent states in a dynamical process. It is assumed that such process behave in order to follow optimal paths in some convenient space of partial information states, called the phase space.

Models are in fact replaced by model schemes. A model scheme is simply a set of properties (justifications) which caracterises a certain class of models.

If the process has $k$ states, represented by sets of partial information ionic formulae $\left(\Phi_{0}, \Phi_{1}, \ldots, \Phi_{k}\right)$ and if $M\left(\Phi_{i}\right)$ is the set of model schemes associated with $\Phi_{i}, M\left(\Phi_{0}\right) \times M\left(\Phi_{1}\right) \times \ldots \times M\left(\Phi_{k}\right)$ is the phase space $\mathbb{P}$ of the process. We then define a regular path as a path $\left(x_{0}, x_{1}, \ldots\right)$ traced through $\mathbb{P}$ such that $\forall i x_{i}$ is minimal in $M\left(\Phi_{i}\right)$ for the cost ordering.

We then adopt the variational principle: Among all paths that it may take through $\mathbb{P}$, the system traces some regular path.

The actual value of the regular path traced through phase space may or may not be known.

The variational principle is used to eliminate incorrect paths. This approach may be seen as providing a (partial) approximation of Grice's maxims in our framework.

The principle of preferring hard conclusions over soft ones also corresponds to the lazy evaluation principle of Piilog, an implementation of logic programming in LPI designed by Rajnovich [5], and amounts to some sort of least effort principle.

Example 1. *Il a lu quelque romans de Balzac, il en a même lu un. The background knowledge $\mathbf{Q}$ for this problem is provided by clauses $(Q 1),(Q 2)$ above. Assume $N=N^{\prime}=5$. 
Let $\Phi_{0}=\{\exists ! n . l u(c, n), \exists \boldsymbol{\gamma} . \star(\boldsymbol{\gamma}$, heap $(c))\}$ represent the first sentence, and let $\Phi_{1}=\Phi_{0} \cup\{l u(c, 1)\}$ represent both sentences. We assume that $\exists ! n . l u(c, n)$ is interpreted as infinite exclusive disjunction $\oplus_{n \in \mathbb{N}} l u(c, n)$.

Let $M\left(\Phi_{0}\right)$ be the set of models schemes of $\Phi_{0}$ together with $\mathbf{Q}$. Set $M\left(\Phi_{0}\right)$ contains infinitely many justification ordering minimal model schemes $m_{n}$, where $n \in \mathbb{N}$ is the number of novels read, given as follows:

$m_{i}=\left\{l u(c, i),+\star l u q(c, 4), \ldots,+\star l u q(c, i),\left.\right|_{\text {soft }}\right.$ heap $\left.(c), \ldots\right\}$ for $i=1, \ldots, 4$.

$m_{5}=\{l u(c, 5), \mid=$ heap $(c), \ldots\}$

$m_{j}=\left\{l u(c, j),+\star l u q(c, 6), \ldots,+\star l u q(c, j), \mid==_{s o f t}\right.$ heap $\left.(c), \ldots\right\}$ for $j>5$.

Pattern $m_{0}=\{l u(c, 0),+\star l u q(c, 4), \ldots,+\star l u q(c, 1),-\star l u q(c, 0), \nVdash$ heap $(c), \ldots\}$

does not fit the bill, since it does not make heap $(c)$ true in a soft sense. In the cost ordering, $m_{1}$ is the maximal element in $M\left(\Phi_{0}\right)$.

Path $\left(x_{0}, x_{1}\right)$ where $x_{0}=m_{1}=\left\{l u(c, 1),+\star l u q(c, 4), \ldots,+\star l u q(c, 1), \mid={ }_{\text {soft }}\right.$ heap $(c), \ldots\}$, and $x_{1}=\{\models h e a p(c), l u(c, 1), \ldots\}$ is the only semantically correct trajectory through $\mathbb{P}$ corresponding to syntactic path $\left(\Phi_{0}, \Phi_{1}\right)$. However, path $\left(x_{0}, x_{1}\right)$ is not regular, since $x_{0} \in M\left(\Phi_{0}\right)$ is not minimal for the cost ordering on $M\left(\Phi_{0}\right)$.

Example 2. *Il a lu quelques romans de Balzac, il n'en a même lu aucun. We now have $\Phi_{0}^{\prime}=\Phi_{0}$ as above, and $\Phi_{1}^{\prime}=\Phi_{0} \cup\{l u(c, 0)\}$. Here, in path $\left(x_{0}, x_{1}\right)$, one must have $x_{0}=m_{n}$ for some $n \neq 0$ as above, in order to make heap $(c)$ true in a soft sense. But $x_{1}$ must make $l u(c, 0)$ true, therefore cannot be an extension of such an $x_{0}$. Therefore, since heap $(c)$ must be false at step $x_{1}$, no trajectory of this system through the phase space makes heap $(c)$ true in a soft sense at step $x_{0}$. In particular, no regular trajectory exists.

\section{Generalization to other vague quantifiers in French}

\subsection{Outline of a general paradigm for scalar implicatures}

The axiomatizations above for quelques comprehend three components:

1. a range corresponding to situations where we must answer with a hard yes. It defines the positive boundary of the predicate luq.

2. a second range corresponding to situations where we must answer with a hard no. It defines the negative boundary of the predicate luq.

3. a third component describing a "soft" domain, corresponding to situations where the sorites axioms must be called upon.

This suggests the following general scheme for vague quantifiers. By $\lambda$-abstraction one may define fixpoint equation

$$
h e a p=\lambda \varphi . \Phi
$$


where $\Phi=\Phi(\varphi, \theta)$ is given by the set of formulae:

$$
\begin{gathered}
\forall k \varphi(k, 0) \rightarrow \neg \text { heap }(k) \\
\exists N \exists N^{\prime} N \leq N^{\prime} . \forall k \forall n N \leq n \leq N^{\prime} \rightarrow \theta(k, n) \\
\forall k \forall n<N \theta(k, n+1) \rightarrow \star(\theta(k, n), \theta(k, n)) \\
\forall k \forall n \geq N^{\prime} \theta(k, n) \rightarrow \star(\theta(k, n+1), \theta(k, n+1))
\end{gathered}
$$

where $\theta$ is a predicate defined by implication:

$$
\theta(k, n):=(\varphi(k, n) \rightarrow \text { heap }(k))
$$

This scheme is quite general. We may retrieve a symmetrical form of the original sorites paradox by choosing predicates $\varphi$, heap and $\theta$ as independent from $k$, and $N=N^{\prime}$ :

$$
\begin{aligned}
& \varphi(0) \rightarrow \neg \text { heap } \\
& \exists N . \forall n . n=N \rightarrow \theta(n) \\
& \forall n<N \theta(n+1) \rightarrow \star(\theta(n), \theta(n)) \\
& \forall n \geq N \theta(n) \rightarrow \star(\theta(n+1), \theta(n+1)) \\
& \forall n . \theta(n) \leftrightarrow(\varphi(n) \rightarrow \text { heap })
\end{aligned}
$$

The original paradox is reached via $p(n):=\theta(n)$. Symmetry is obtained via the addition of an upward induction sorites axiom (30). Clearly, if the number of grains of sand is equal to the number of particles of the universe, then we may conclude that we still have a heap of sand only in a weak sense.

\subsection{Application to other vague quantifiers}

Preliminary investigations show that the above approach applies to other vague quantifiers in French, modulo some variations of the topology of the argumentation scale. It turns out that every vague quantifier is characterized by

1. a positive boundary $B^{+}$.

2. a negative boundary $B^{-}$

3. one or two sorites induction axioms ranging over intervals $S^{\uparrow}, S^{\downarrow}$.

For example, for the quelques example discussed above, $B^{-}=\{0\}, B^{+}=$ $\left\{N, N+1, \ldots, N^{\prime}\right\}, S^{\downarrow}=\{1, \ldots, N-1\}, S^{\uparrow}=\left\{N^{\prime}+1, \ldots, T\right\}$, where $T$ is the total number of Balzac novels. And for the sorites paradox, $B^{+}=\{N\}$, $B^{-}=\{0\}, S^{\downarrow}=\{1, \ldots, N-1\}$,

Examples of such topologies are as follows.

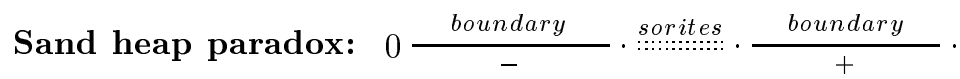

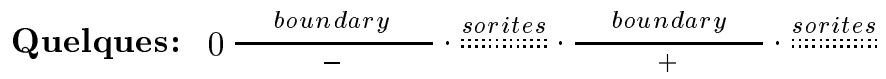




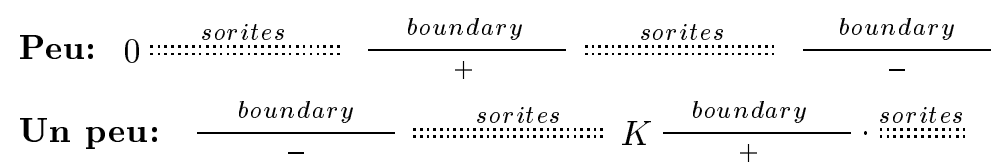

Beaucoup: $0 \frac{\text { boundary }}{-} \cdot \begin{gathered}\text { sorites } \\ -\end{gathered} \quad \frac{\text { boundary }}{+}$

Pas quelques: 0 sorites $:$ boundary sorites $_{+}^{\text {houndary }}$

Thus the respective patterns of these examples over the alphabet $\left\{B^{+}, B^{-}, \uparrow, \downarrow\right\}$ may be summarized as follows:

\begin{tabular}{|l|c|}
\hline quantifier & pattern \\
\hline sorites paradox & $B^{-} \downarrow B^{+}$ \\
\hline quelques & $B^{-} \downarrow B^{+} \uparrow$ \\
\hline peu & $\downarrow B^{+} \uparrow B^{-}$ \\
\hline un peu & $B^{-} \downarrow B^{+} \uparrow$ \\
\hline beaucoup & $B^{-} \downarrow B^{+}$ \\
\hline pas quelques & $\downarrow B^{+} \uparrow B^{-}$ \\
\hline
\end{tabular}

Let us examine some differences between words like peu, un peu and quelques. Intuitiveley, peu and un peu seem quite similar, but they differ in their argumentative orientation, as shown by:

$$
\text { Il a lu peu de livres de Balzac DONC il ne connaît pas bien Balzac }
$$

(he read few books of Balzac, therefore he does not know Balzac well) versus:

$$
\text { Il en a lu un peu DONC il connaît (un peu) Balzac }
$$

(he read a few, therefore he knows (a little) about Balzac)

By (32), we mean that the person has read less than $K$ books, for some rather low $K$. The set of formulae corresponding to peu is:

$$
\begin{aligned}
& \quad \forall k \varphi(k, T) \rightarrow \neg h \operatorname{eap}(k) \\
& \exists K \forall k \forall n .1 \leq n \leq K \rightarrow \theta(k, n) \\
& \quad \forall k \forall n<1 . \theta(k, n+1) \rightarrow \star(\theta(k, n), \theta(k, n)) \\
& \quad \forall k \forall n \geq K . \theta(k, n) \rightarrow \star(\theta(k, n+1), \theta(k, n+1))
\end{aligned}
$$

This corresponds to the topological scale above: the negative boundary is $B^{-}=$ $\{T\}$, where $T$ is the total number of books, the positive boundary is therefore the opposite one, provided by small values: $B^{+}=\{1,2 \ldots, K\}$. Sorites are used for values less than 1 and values greater than $K$. Because 0 is on the positive orientation, it will be possible to assert:

$$
\text { Il a lu peu de livres de Balzac... il n'en a même lu aucun }
$$

Now, by (33), we mean that the person has read more than $K$ books ( $K$ small) but less than a certain $K^{\prime}$. The set of formulae is then the same as for quelques, 
but a comparison of the two words (as they could appear in the same discourse or uttered by the same locutor etc.), shows that $K$ is systematically lower than the $N$ used in the set of formulae for quelques in the same context.

The case of beaucoup is similar, using the same scheme as for un peu and quelques, but different values for $N, N^{\prime}$. If we associate with each word $w$ of this family its own interval of positive "hard" conclusions: $B^{+}=B_{w}^{+}=\left\{N_{w}, N_{w}+1, \ldots, N_{w}^{\prime}\right\}=$ $\left[N_{w}, N_{w}^{\prime}\right]$, we have:

$$
\begin{aligned}
& N_{\text {unpeu }}<N_{\text {quelques }}<N_{\text {beaucoup }} \\
& N_{\text {unpeu }}^{\prime}<N_{\text {quelques }}^{\prime}<N_{\text {beaucoup }}^{\prime}
\end{aligned}
$$

but the scales associated with these three words have the same orientation (different from the one of peu, and pas...quelques). We can actually represent all these scales as subscales of a general one, in such a way that the following fragments of discourse are made possible:

Il a lu un peu de Balzac, il en a lu quelques romans

$$
\text { Il a lu un peu de Balzac, il en a même lu beaucoup }
$$

$$
\text { Il a lu quelques romans de Balzac, il en a même lu beaucoup }
$$

Let us also observe that from

$$
\text { Il a lu beaucoup de romans de Balzac }
$$

it is possible to draw the conclusion:

$$
\text { Il a lu quelques romans de Balzac }
$$

but as a "hard" consequence, by following the same reasoning as in section 4 for proving that il n'a pas lu quelques romans de Balzac may be deduced from il a lu quelques romans de Balzac.

\subsection{Further applications}

This also generalizes to scalable adjectives, etc. Statement The Pathfinder expedition to Mars was expensive corresponds to scheme

$$
0 \frac{\text { boundary }}{-} \cdot \text { sorites } \frac{\text { boundary }}{+}
$$

where $B^{-}$corresponds to (depending on the scale) "the expedition was actually not expensive" and $B^{+}$corresponds to "the expedition was actually expensive." 
This regularity suggests sharpening up as follows our approach to the formalization for vague quantifiers in French.

An argumentation scale is a linearly ordered set. Such a linear order may or may not have a minimal (resp. maximal) element. For the purpose of the current discussion, we assume that the ordering is discrete, i.e. that it may embedded into $\mathbb{Z}$.

A general view of vague quantifiers from the argumentation point of view is as follows. Here the definiendum is predicate heap, and the definiens is predicate $\varphi$.

Let $\mathbb{A}$ be an argumentation scale. Let $\varphi(k, n)$ be some given predicate. $\exists B^{+}, B^{-}, S^{\uparrow}, S^{\downarrow} \subseteq \mathbb{A}$ such that

1. $\left\{B^{+}, B^{-}, S^{\uparrow}, S^{\downarrow}\right\}$ is a partition of argumentation scale $\mathbb{A}$.

2. $\forall k \forall n \in B^{+} \varphi(k, n) \rightarrow h e a p(k)$ (i.e. $\forall k \forall n \in B^{+} \theta(k, n)$.)

3. $\forall k \forall n \in B^{-} \varphi(k, n) \rightarrow \neg$ heap $(k)$

4. $\forall k \forall(n+1) \in S^{\uparrow} \theta(k, n) \rightarrow \star(\theta(k, n+1), \theta(k, n+1))$

5. $\forall k \forall n \in S^{\downarrow} \theta(k, n+1) \rightarrow \star(\theta(k, n), \theta(k, n))$

Furthermore, $B^{+}, B^{-}, S^{\uparrow}, S^{\downarrow}$ must be intervals, and thus generate a topology, in the intuitive sense.

Whence, if $\mu h . \Phi(h)$ designates the least defined predicate satisfying $\Phi(h)$, it seem that a general scheme encompassing all these case is given by definition:

$$
\begin{aligned}
q=\mu h . \exists B^{+}, B^{-}, S^{\uparrow}, S^{\downarrow} \subseteq \mathbb{A} & . \mathbb{A}=B^{+} \uplus B^{-} \uplus S^{\uparrow} \uplus S^{\downarrow} \\
& \forall k \forall n \in B^{+} \theta(k, n) \\
& \forall k \forall n \in B^{-} \varphi(k, n) \rightarrow \neg h(k) \\
& \forall k \forall(n+1) \in S^{\uparrow} \theta(k, n) \rightarrow \star(\theta(k, n+1), \theta(k, n+1)) \\
& \forall k \forall n \in S^{\downarrow} \theta(k, n+1) \rightarrow \star(\theta(k, n), \theta(k, n))
\end{aligned}
$$

where one defines locally $\theta(k, n):=(\varphi(k, n) \rightarrow h(k))$. The variations between different vague quantifers are accounted for in this definition by using as input different partitions of argumentation scale $\mathbb{A}$.

\section{Conclusion}

We have attempted to take into account some earlier analyses, particularly by $\mathrm{O}$. Ducrot [2], concerning some very sensitive discourse items which very frequently occur in our everyday language. Such phenomena are very often dealt with by calling up Grice's maxims. It is true that classical pragmatics may be efficiently applied here, but we regret that Grice's maxims have currently no adequate formalisation. This is the reason why we attempt to provide formal tools capable at least to emulate what they actually can do.

Our approach is distinct from two other ones which have been popular in the past:

- the non monotonic logical approach

- the "fuzzy sets" approach 
It is distinct from the first one because, as has been said in Section 2, it is not limited to the search of consistent sets of beliefs, it allows computations step by step and thus provides a way of representing dynamic processes. It is distinct from the second one because, besides the fact that it does not use any kind of "spurious" quantification, the undetermination is not seen here as some de re property of the (ontological) entities themselves, but as a way of apprehending reality through language.

Concerning the latter point, it must be said that the true appropriate way of formulating the issues here presented is probably dialogical. We must think of two speakers engaged in a conversation, who try to agree on the sense of words and their applicability to a given situation. Such a conversation could be like the following:

A: Peter read some novels by Balzac

B: what do you mean by "some"? five? six?

A: around five

B: actually, I know that Peter read three novels, do you still accept he read "some"?

The appropriateness of "some" thus results from conventions that locutors state themselves, and from some kind of negociation process according to which they finally agree whether they agree on certain justifications. This is very close to the Wittgensteinian notion of language game [6], and calls for a theoretical framework where word meanings would be negociated according to this pragmatical conception of language. When we reflect on language, we perceive that not only discourse items like quelques, peu, un peu, beaucoup etc. are "vague" but ... almost every word!

\section{References}

1. G. Chierchia and S. McConnell-Ginet. Meaning and grammar. MIT Press, 2001.

2. O. Ducrot. Les échelles argumentatives. Editions de Minuit, 1980.

3. L. Horn. On the semantic properties of logical operators in English. $\mathrm{PhD}$ thesis, 1972.

4. M. A. Nait Abdallah. The logic of partial information. EATCS Research Monographs in Theoretical Computer Science. Spinger Verlag, 1995.

5. J.J. Rajnovich. Piilog: Partial information ionic logic programming. PhD thesis, Department of Computer Science, University of Western Ontario, 2000.

6. L. Wittgenstein Philosophical Investigations edited by G.E.M. Anscombe and R. Rhees. Oxford: Basil Blackwell, 1951. 